\title{
Improved Surface and Tropospheric Temperatures Determined Using Only Shortwave Channels: The AIRS Science Team Version-6 Retrieval Algorithm
}

\author{
Joel Susskind $^{1}$, John Blaisdell ${ }^{2}$, and Lena Iredell ${ }^{2}$ \\ ${ }^{1}$ NASA Goddard Space Flight Center, Greenbelt, MD, USA 20771 \\ ${ }^{2}$ SAIC, NASA Goddard Space Flight Center, Greenbelt, MD, USA 20771
}

\begin{abstract}
The Goddard DISC has generated products derived from AIRS/AMSU-A observations, starting from September 2002 when the AIRS instrument became stable, using the AIRS Science Team Version-5 retrieval algorithm. The AIRS Science Team Version-6 retrieval algorithm will be finalized in September 2011. This paper describes some of the significant improvements contained in the Version-6 retrieval algorithm, compared to that used in Version-5, with an emphasis on the improvement of atmospheric temperature profiles, ocean and land surface skin temperatures, and ocean and land surface spectral emissivities. AIRS contains 2378 spectral channels covering portions of the spectral region $650 \mathrm{~cm}^{-1}(15.38 \mu \mathrm{m})-2665 \mathrm{~cm}^{-1}(3.752 \mu \mathrm{m})$. These spectral regions contain significant absorption features from two $\mathrm{CO}_{2}$ absorption bands, the $15 \mu \mathrm{m}$ (longwave) $\mathrm{CO}_{2}$ band, and the $4.3 \mu \mathrm{m}$ (shortwave) $\mathrm{CO}_{2}$ absorption band. There are also two atmospheric window regions, the $12 \mu \mathrm{m}-8 \mu \mathrm{m}$ (longwave) window, and the $4.17 \mu \mathrm{m}-3.75 \mu \mathrm{m}$ (shortwave) window. Historically, determination of surface and atmospheric temperatures from satellite observations was performed using primarily observations in the longwave window and $\mathrm{CO}_{2}$ absorption regions. According to cloud clearing theory, more accurate soundings of both surface skin and atmospheric temperatures can be obtained under partial cloud cover conditions if one uses observations in longwave channels to determine coefficients which generate cloud cleared radiances $\widehat{\boldsymbol{R}}_{\boldsymbol{i}}$ for all channels, and uses $\widehat{\boldsymbol{R}}_{\boldsymbol{i}}$ only from shortwave channels in the determination of surface and atmospheric temperatures. This procedure is now being used in the AIRS Version-6 Retrieval Algorithm. Results are presented for both daytime and nighttime conditions showing improved Version-6 surface and atmospheric soundings under partial cloud cover.
\end{abstract}

Keywords: AIRS/AMSU, high spectral resolution IR sounders, retrieval methodology, IR sounding in cloudy conditions, cloud cleared radiances, Quality Control.

\section{INTRODUCTION}

AIRS was launched on EOS Aqua in May 2002, together with AMSU-A and HSB, to form a next generation polar orbiting infrared and microwave atmospheric sounding system ${ }^{1}$. AIRS is a grating spectrometer with a number of linear arrays of detectors with each detector sensitive to outgoing radiation in a characteristic frequency $v_{i}$ with a spectral band pass $\Delta v_{i}$ of roughly $v_{i} / 1200$. AIRS contains 2378 spectral channels covering portions of the spectral region $650 \mathrm{~cm}^{-1}$ $(15.38 \mu \mathrm{m})-2665 \mathrm{~cm}^{-1}(3.752 \mu \mathrm{m})$. The spectral sampling interval (except for the existence of a few gaps) is $v_{i} / 2400$, giving two AIRS channels per spectral half width. AIRS is accompanied by the temperature sounding $60 \mathrm{GHz}$ microwave instrument AMSU-A. There is a 3x3 array of AIRS footprints within a given AMSU-A footprint, with spatial resolutions of $13 \mathrm{~km}$ and $45 \mathrm{~km}$ at nadir viewing respectively. HSB, a $183 \mathrm{GHz}$ water vapor sounding microwave instrument, failed early in the mission.

The Goddard DISC has generated products derived from AIRS/AMSU-A observations, starting from September 2002 when the AIRS instrument became stable, using the AIRS Science Team Version- 5 retrieval algorithm. ${ }^{2}$ The AIRS Science Team Version-6 retrieval algorithm will be finalized in September 2011, and the Goddard DISC will start processing AIRS/AMSU data as well as reprocessing all AIRS/AMSU data using the AIRS Science Team Version-6 retrieval algorithm in early 2012. The Version-6 retrieval observations will be run both in an AIRS/AMSU mode as well as in an "AIRS Only" mode designed as a back-up system to be used in the event that the AMSU-A instrument should continue to degrade, as has been the case up to the present. This paper describes some of the significant improvements 
contained in the Version-6 retrieval algorithm, compared to that used in Version-5, with an emphasis on the improvement of atmospheric temperature profiles, ocean and land surface skin temperatures, and ocean and land surface spectral emissivities. The results shown in this paper will be referred to as "Version- 6 ". There may be some small differences in the final Version-6 product from those shown in this paper. AIRS Version-6 retrievals also contain significant improvements in trace gas vertical profiles, cloud products, and OLR and Clear Sky OLR products. These improvements are not discussed in this paper.

The theoretical approach used to analyze AIRS/AMSU data in the presence of clouds in the AIRS Science Team Version-3 at-launch algorithm, and that used in the Version-4 post-launch algorithm, have been published previously ${ }^{3,4}$. The fundamental approach used in the analysis of AIRS/AMSU observations is the generation of clear column radiances for each AIRS channel $i, \hat{R}_{i}$, which are derived products representing the radiance channel $i$ would have seen if the entire 3x3 AIRS Field of Regard (FOR) were cloud free. The retrieved geophysical state $X$ is subsequently determined which, when substituted in a Radiative Transfer Algorithm (RTA), generates an ensemble of computed radiances $R_{i}(X)$ which are consistent with $\hat{R}_{i}$ for those channels i used in the determination of $X$. The AIRS Science Team Version-5 algorithm ${ }^{2}$ contained three significant improvements over Version-4. Improved physics in Version-5 allowed for use of AIRS clear column radiances $\hat{R}_{i}$ in the entire $4.3 \mu \mathrm{m} \mathrm{CO}$ absorption band in the retrieval of temperature profiles $\mathrm{T}(\mathrm{p})$ during both day and night. This is consistent with cloud-clearing theory ${ }^{5,6}$ which says that to achieve the best results in more stressing cloud conditions, longwave channels sensitive to cloud contamination should be used only in the generation of clear column radiances and not for sounding purposes. In Version- $5^{2}$, tropospheric sounding $15 \mu \mathrm{m} \mathrm{CO}_{2}$ observations were used only in the derivation of coefficients which allow for the generation of clear column radiances $\hat{R}_{i}$ for all channels. This new approach allowed for the generation of accurate Quality Controlled values of $\hat{R}_{i}$ and $T(p)$ under more stressing cloud conditions than was achievable in Version-4. Secondly, Version-5 contained a new methodology to provide accurate case-by-case error estimates for retrieved geophysical parameters and for channel-by-channel clear column radiances. Thresholds of these error estimates were used in the new approach for Quality Control. Finally, Version-5 contained for the first time an approach to provide AIRS soundings in partially cloudy conditions that does not require use of any microwave data. This new AIRS Only sounding methodology, referred to as AIRS Version-5 AO, was developed as a backup to AIRS Version-5 should the AMSU-A instrument fail. Susskind et al. ${ }^{2}$ show that Version-5 AIRS Only soundings are only slightly degraded from the AIRS/AMSU soundings, even at large fractional cloud cover. Version-5 AIRS Only retrievals were not run routinely at the Goddard DISC however.

The AIRS Version-6 retrieval algorithm has further significant advances over Version-5. The basic theoretical approach used in Version-6 to analyze AIRS/AMSU data is very similar to Version-5 with one major exception. As in Version-5, the generation of cloud cleared radiances $\hat{R}_{i}$ for all channels is performed using observed radiances $R_{i}$ for longwave 15 $\mu \mathrm{m}$ and $11 \mu \mathrm{m}$ channels. Tropospheric temperature profiles were retrieved in Version-5 using only the AIRS shortwave $4.2 \mu \mathrm{m} \mathrm{CO} 2$ channels, but surface skin temperature was retrieved simultaneously with surface spectral emissivity and bidirectional reflectance using observations both in the longwave 8-12 $\mu \mathrm{m}$ window region and in the shortwave $4.0 \mu \mathrm{m}-$ $3.76 \mu \mathrm{m}$ window region. In Version-6, only window observations in the shortwave window region, $4.0 \mu \mathrm{m}-3.76 \mu \mathrm{m}$ are used to simultaneously determine surface skin temperatures along with shortwave surface spectral emissivities and surface bi-directional reflectance. The current use of only shortwave AIRS channels in the retrieval of both atmospheric and surface parameters has resulted in significant improvement in the ability to obtain both accurate temperature profiles and surface skin temperatures under more stressing partial cloud cover conditions. The Version-6 retrieved algorithm also has significant improvements over Version-5 in its Quality Control methodology as well as in the generation of the start-up state $X^{o}$ used to initialize the cloud clearing and retrieval procedure.

\section{STEPS IN THE VERSION-6 RETRIEVAL ALGORITHM}

Retrievals of all geophysical parameters are physically based and represent states $X_{j, c}$ determined for case c that best match a set of clear column radiances $\hat{R}_{i, c}$ for the subset of AIRS channels $i$ used in the retrieval process. Retrievals of geophysical parameters are performed sequentially, that is, only a subset of the geophysical parameters within the state $X_{j}$ is modified from that of the incoming state $X_{j}^{0}$ in a given step. A GCM forecast is not used in any way in the retrieval procedure, except for the forecasted surface pressure $\mathrm{p}_{\text {surf }}$, which is used as the lower pressure boundary when computing expected radiances $R(X)$ for a given geophysical state $X_{j}$. 
In Version- $5^{2}$, the steps in the physical retrieval process are as follows: A regression-based start-up procedure is used to generate the initial state $X^{o}$. Initial clear column radiances $\hat{R}_{k}^{0}$ are generated for all channels $k$ using the initial state $X^{o}$ and an ensemble of cloud clearing channels $\mathrm{k}^{3}$. The state $X^{o}$ is also used as the initial guess to the physical retrieval process in which AIRS/AMSU (or AIRS Only) observations are used to retrieve: a) surface skin temperature, surface spectral emissivity and surface bi-directional reflectance of solar radiation; b) atmospheric temperature profile; c) atmospheric moisture profile; d) atmospheric ozone profile; e) atmospheric $\mathrm{CO}$ profile; f) atmospheric $\mathrm{CH}_{4}$ profile; and g) cloud properties and OLR. These steps are done sequentially, solving only for the variables to be determined in each retrieval step and using previously determined variables as fixed but with an appropriate uncertainty attached to them which is accounted for in the channel noise covariance matrix N. The objective in each step (a-f) is to find solutions which best match $\hat{R}_{i}$ for the subset of channels selected for use in that step, bearing in mind the channel noise estimates. Steps a-f are ordered so as to allow for selection of channels in each step which are primarily sensitive to variables to be determined in that step or determined in a previous step, and relatively insensitive to other parameters. Separation of the problem in this manner also allows for the problem in each step to be made as linear as possible. Step g is performed after the surface and atmospheric conditions have been determined using the observed radiances $R_{i}$.

In Version-6, there is a slight modification to the sequence of steps used in Version-5, as there is a new step performed in the retrieval sequence. In Version-5, step a) used channels in both the longwave and shortwave window regions and simultaneously solved for surface skin temperature $T_{s}$, shortwave surface spectral emissivity $\varepsilon_{s w}(v)$ and surface spectral bi-directional reflectance $\rho_{s w}(v)$, and longwave surface spectral emissivity $\varepsilon_{\ell w}(v)$. In Version-6, only shortwave window channels are used in this retrieval step to simultaneously determine $T_{s}, \varepsilon_{s w}(v)$, and $\rho_{s w}(v)$. The longwave surface spectral emissivity $\varepsilon_{\ell w}(v)$ is now solved for in a subsequent step using only channels in the longwave window spectral region. This new step is performed after the humidity profile retrieval step because longwave window radiances can be very sensitive to the amount of atmospheric water vapor. The start-up state used in Version-5 is based on a linear regression expressing the relationship between $\hat{R}_{i}$ and the geophysical state $X^{0}$. Version- 6 uses a more advanced neuralnetwork approach to generate $X^{0}$ from the observed radiances $R_{i} \cdot{ }^{7,8}$ The steps used in the Version-6 AO algorithm are otherwise identical but no AMSU-A observations are used in the physical retrieval process or in the generation of the initial state $X_{i}^{0}$.

\section{CHANNELS AND FUNCTIONS USED IN DIFFERENT STEPS OF VERSION-6}

Figure 1 shows a typical AIRS cloud free brightness temperature spectrum and includes the channels used in Version-6 and Version-6 AO for cloud clearing, as well as in each of the different steps of the AIRS physical retrieval algorithm. These channels are described below.

\subsection{Cloud clearing and temperature profile retrieval}

Following cloud-clearing theory, coefficients needed to generate clear column radiances for all channels are determined using observations in 56 longwave channels, ranging from $701 \mathrm{~cm}^{-1}-1072 \mathrm{~cm}^{-1}$, which are shown in yellow in Figure 1 . These same channels are also the ones used in the cloud parameter retrieval step ${ }^{3}$. The temperature profile retrieval step uses 37 channels between $2358 \mathrm{~cm}^{-1}$ and $2395 \mathrm{~cm}^{-1}$ that are sensitive to both stratospheric and tropospheric temperatures, as well as 60 stratospheric sounding channels between $662 \mathrm{~cm}^{-1}$ and $713 \mathrm{~cm}^{-1}$ that are not sensitive to cloud contamination. These channels are shown in red in Figure 1. Version-6 also includes 24 additional channels in the temperature profile retrieval step between $2396 \mathrm{~cm}^{-1}$ and $2418 \mathrm{~cm}^{-1}$, shown in dark blue, that are used in both the temperature profile and the surface parameter retrieval steps. Version-6 includes AMSU-A channels 3, 6 and 8-14 in the temperature profile retrieval step as well, while Version-6 AO does not. Versions-4 and -5 also included AMSU-A channels 4 and 5 in the temperature profile retrieval step, but those channels became noisy in 2007 and are no longer used. AMSU-A channel 7 was noisy at launch and was never used in any step of the retrieval process. As discussed previously, Version-6 has separate steps to generate surface skin temperature, and subsequently to generate longwave surface spectral emissivity. 


\subsection{Surface skin temperature and longwave spectral emissivity retrievals}

The surface skin temperature retrieval step uses 37 channels between $2420 \mathrm{~cm}^{-1}$ and $2664 \mathrm{~cm}^{-1}$, shown in light blue in Figure 1, along with those shown in dark blue which are also used in the temperature profile retrieval step. These 64 channels are used to determine the surface skin temperature simultaneously with 4 independent pieces of information about surface shortwave spectral emissivity and 4 additional independent pieces of information of shortwave surface bidirectional reflectance during the day. Therefore the skin temperature retrieval steps solves for nine unknowns during the day and five unknowns at night.

Surface longwave spectral emissivity is determined using 79 channels, between $758 \mathrm{~cm}^{-1}$ and $1250 \mathrm{~cm}^{-1}$, which are shown in purple in Figure 1. In this step, in which 3 longwave emissivity perturbation functions are solved for, $T_{s}$ is held fixed at the value determined from the previous skin temperature retrieval step.

The initial guess for surface spectral emissivity in both retrieval steps, $\varepsilon_{v}^{0}$, is set equal to the values found in the AIRS Science Team ocean emissivity model over non-frozen ocean, and over land and frozen ocean, $\varepsilon_{v}^{0}$ is set equal to values coming from the $1^{\circ} \times 1^{\circ}$ monthly mean MODIS Science Team surface spectral emissivity data set.

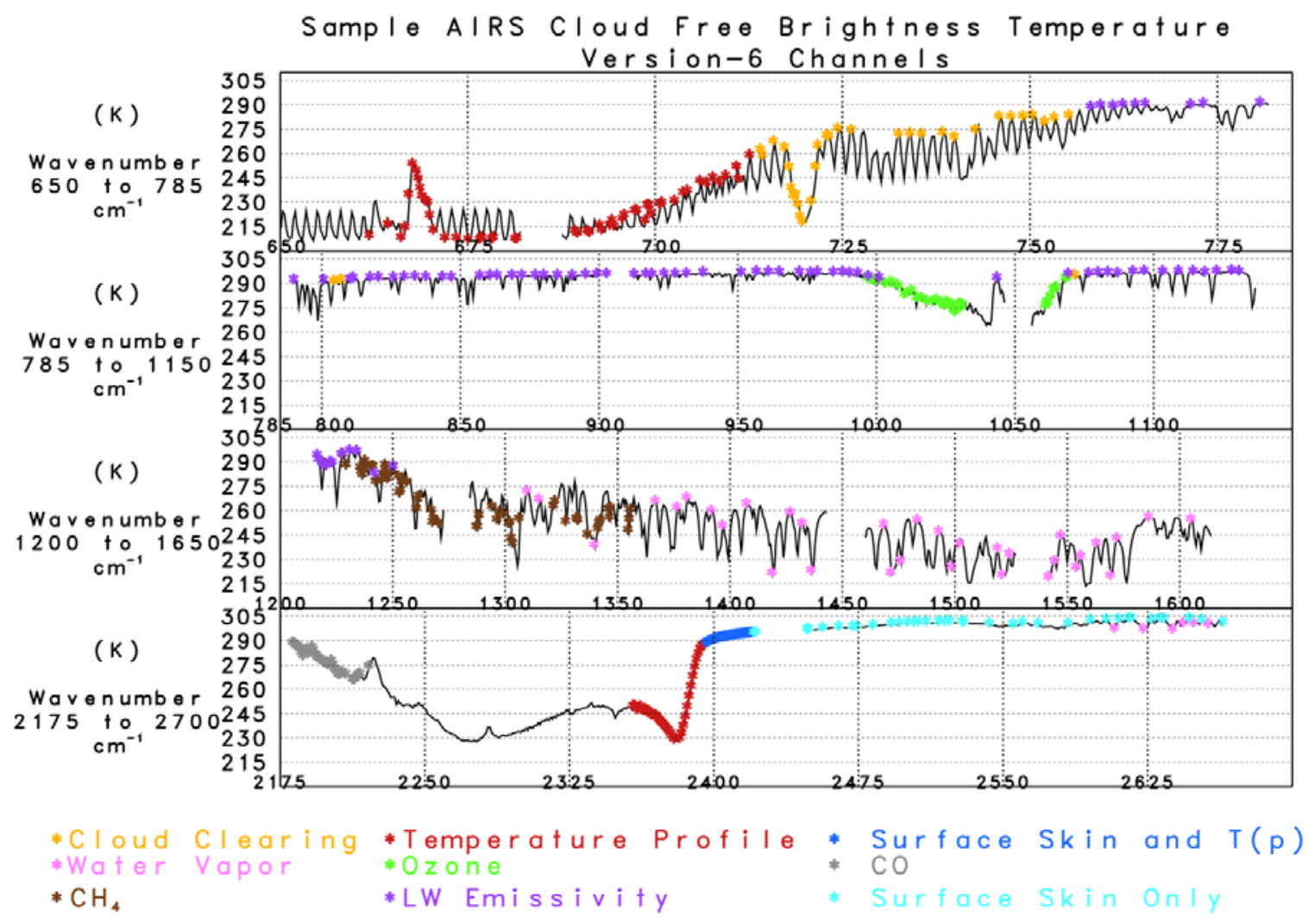

Fig. 1

\subsection{Constituent profile retrievals}

As in Version-5, constituent profile retrievals are performed in separate subsequent steps, each having their own set of channels and functions ${ }^{2,3}$. Figure 1 shows in different colors the Version- 6 channels used in each of these retrieval steps. The $q(p)$ retrieval (pink stars) uses 48 channels in the spectral ranges $1310 \mathrm{~cm}^{-1}$ to $1605 \mathrm{~cm}^{-1}$ and $2608 \mathrm{~cm}^{-1}$ to $2656 \mathrm{~cm}^{-1}$; the $O_{3}(p)$ retrieval (green stars) uses 41 channels between $997 \mathrm{~cm}^{-1}$ and $1069 \mathrm{~cm}^{-1}$; the $C O(p)$ retrieval (gray stars) uses 36 channels between $2181 \mathrm{~cm}^{-1}$ and $2221 \mathrm{~cm}^{-1}$; and the $\mathrm{CH}_{4}(p)$ retrieval (brown stars) uses 60 channels between $1220 \mathrm{~cm}^{-1}$ and $1356 \mathrm{~cm}^{-1}$. 


\section{COMPARISON OF QUALITY CONTROLLED VERSION-6 AND VERSION-6 AO TEMPERATURE AND EMISSIVITY RETRIEVALS WITH THOSE OF VERSION-5}

The subsequent discussion in this paper deals only with Version-6 and Version-6 AO results of Quality Controlled temperature profile and surface skin parameters. After a discussion of Version-6 Quality Control methodology, Version6 and Version-6 AO results are compared to those obtained using Version-5. All results shown are based on average statistics over seven days run in different seasons and different years ranging from September 6, 2002 through May 20, 2010.

\subsection{Quality controlled temperature profiles}

The basic approach to temperature profile Quality Control used in Version-6 is analogous to that used in Version-5 with two important differences. The AIRS Version-5 Science Team retrieval algorithm introduced the concept of assigning to each temperature profile $T(p)$ a pressure, $p_{\text {best }}$, down to which $T(p)$ is considered to be of highest quality. Version-5 generated and used case-by-case level-by-level error estimates for temperature profiles at pressure $p_{i} \Delta T\left(p_{i}\right)^{2}$, to determine the case-by-case characteristic pressure $p_{\text {best }}$. Roughly $15 \%$ of all cases, particularly those retrieved under more than $90 \%$ fractional cloud cover, were considered not to be suitable for use in scientific research and were rejected outright. In Version-5, all accepted profiles are assigned to have highest quality down to at least $70 \mathrm{mb}$. The characteristic pressure $p_{\text {best }}$ was defined as the largest pressure (somewhere between $70 \mathrm{mb}$ and $p_{\text {surf }}$ ) at which the error estimate in each of the next three pressure levels is not greater than a pressure dependent error estimate threshold $\delta T(p)$. Use of larger values of $\delta T(p)$ results in the acceptance of more cases at a given pressure, with increased spatial coverage that is important for use in climate applications, but with a degradation of overall accuracy. Tightening $\delta T\left(p_{i}\right)$ thresholds results in a lower percentage yield as a function of pressure, but with increased overall accuracy that is important for use in data assimilation experiments.

Pressure dependent thresholds are determined from a set of three threshold parameters $\delta T_{70}, \delta T_{\text {mid }}$, and $\delta T_{\text {surf }}$, representative of error thresholds for $T(p)$ at $p=70 \mathrm{mb}$, at $p=p_{\text {surf }} / 2$, and at $p=p_{\text {surf }}$ where $p_{\text {surf }}$ is the surface pressure. The thresholds $\delta T_{i}$ at intermediate pressures are linearly interpolated in $\log p$ between the given values. It was found advantageous in Version- 5 to have separate error thresholds for non-frozen ocean on the one hand, and land and ice on the other. Table 1 shows the Standard Version-5 thresholds for both non-frozen ocean (called Ocean) and other than nonfrozen ocean (called Land). These Standard thresholds are the ones used at the Goddard DISC in the Version-5 Quality Control, and were optimized to provide a trade-off between reasonable spatial coverage for climate purposes on the one hand, and accuracy for data assimilation purposes on the other. Subsequent research has shown that use of tighter Quality Control than the Standard thresholds used in Version-5 performs better for data assimilation purposes, while looser Quality Control with better spatial coverage, would perform better for climate purposes. Table 1 includes values of a tight set of thresholds that has been shown to perform better in data assimilation experiments. In these experiments, forecasts performed from analyses which assimilated AIRS Quality Controlled temperature soundings down to $p_{\text {best }}$ defined using theTight thresholds were significantly improved compared to analogous experiments in which AIRS Quality Controlled temperature profiles were assimilated down to $p_{\text {best }}$ as defined by the Standard thresholds.

Table 1

Temperature Profile Thresholds (K)

$\begin{array}{ccccccc} & & \begin{array}{c}\text { Ocean } \\ \delta T_{\text {mid }}\end{array} & \delta T_{\text {surf }} & \delta T_{70} & \delta T_{\text {mid }} & \delta T_{\text {surf }} \\ & \delta T_{70} & & & & & \\ \text { Standard } & 1.75 & 1.25 & 2.25 & 2.25 & 2.0 & 2.0 \\ \text { Tight } & 1.75 & 0.75 & 1.75 & 1.75 & 0.75 & 1.75\end{array}$

Figures $2 \mathrm{a}$ and $2 \mathrm{~b}$ show the seven day average global mean statistics for the percent of accepted cases as a function of pressure, and the RMS difference of these retrievals from colocated values of the ECMWF analyses, which we will call RMS errors. Average seven day percent yields and RMS errors of Version-5 retrievals using Standard Quality Control are shown in dashed red and Tight Quality Control are shown in solid red. Average seven day percent yields of 
Version-5 global accepted retrievals using either set of thresholds is about $84 \%$ at $30 \mathrm{mb}$ because roughly $16 \%$ of Version-5 retrievals have been "rejected". The percent yields of Version- 5 accepted retrievals down to $p_{\text {best }}$ begins to diverge at $70 \mathrm{mb}$ when the Quality Control procedure starts to be applied. Figures 2a and 2b show that tightening Quality Control thresholds results in cases having decreased percentage yields and correspondingly increased accuracy (smaller errors) for the accepted cases. It is noteworthy that using Tight Quality Control resulted in RMS errors on the order of $1 \mathrm{~K}$ from $300 \mathrm{mb}$ down to the surface. Experience has shown that this accuracy is needed for the approach of assimilation of Quality Controlled AIRS temperature profiles to perform optimally. Global mean yields of Version-5 Quality Controlled temperature profiles using Standard thresholds are roughly $70 \%$ at $500 \mathrm{mb}$, compared to $34 \%$ using Tight thresholds, and $40 \%$ at the surface compared to $28 \%$, with correspondingly larger RMS differences from ECMWF truth on the order of 0.3K. Use of looser Quality Controlled thresholds would have resulted in a larger ensemble of accepted cases, but with larger RMS errors.

Analogous statistics for Version-6 retrievals and Version-6 AO retrievals are shown in black and green in Figures 2a and $2 \mathrm{~b}$, respectively. Version-6 Quality Control procedures are analogous to those used in Version-5, with two main differences. In Version-6 all retrievals are considered acceptable for scientific use unless the retrieval procedure fails at some point. This happens roughly $1 \%$ of the time. Because accepted cases may be overcast as high as $70 \mathrm{mb}$, Version- 6 Quality Control starts to be applied at $30 \mathrm{mb}$ instead of $70 \mathrm{mb}$ as done in Version-5. The second difference is in the use of two separate sets of Quality Control thresholds. Bearing in mind the experience using the AIRS Version-5 Quality Control procedure in which Standard Quality Control thresholds were too loose for optimal use in data assimilations on the one hand, and too tight for optimal climate application on the other, Version-6 uses separate thresholds for data assimilation purposes and for climate purposes. Data assimilation thresholds are designed to provide RMS errors on the order of $1 \mathrm{~K}$ throughout the troposphere, which define $p_{\text {best }}$. Results using data assimilation (DA) thresholds, giving statistics of yield and accuracy of accepted retrievals down to $p_{\text {best }}$, are shown by the solid black and green lines in Figure 2. A looser set of climate thresholds are used to generate $p_{\text {good }}$, the pressure down to which the retrievals are considered useful for climate purposes. Statistics down to $p_{\text {good }}$ are shown as dashed black and green lines in Figure 2.

Figure 2 shows that percent yields of Version-6 and Version-6 AO accepted retrievals using DA Quality Control are considerably higher than those of Version-5 using Tight Quality Control at all pressure levels, and RMS errors of these retrievals are better than or equal to those of Version-5 at all levels. Assimilation of Version-6 retrievals using DA Quality Control should lead to a further improvement in forecast skill over that obtained assimilating Version-5 retrievals using Tight Quality Control because of the increased yield of high accuracy retrievals and the ability to assimilate accurate soundings above the clouds in overcast situations and storms. Performance of Version-6 AO retrievals using DA Quality Control is only slightly poorer than that of Version-6, primarily in the sense of a somewhat reduced yield with comparable accuracy.

The dashed black and green lines in Figure 2 show that Version-6 and Version-6 AO yields using Climate Quality Control thresholds are considerably higher than those using Version-5 with Standard thresholds and have comparable or better accuracy down to about $700 \mathrm{mb}$, with somewhat degraded, but still very good, accuracy down to the surface. Global yields of Version-6 climate quality retrievals are better than $95 \%$ at $500 \mathrm{mb}$ and roughly $75 \%$ at the surface. Retrievals using Climate Quality Control are the ones included in the gridded Level-3 products produced at the Goddard DISC for distribution to the general public. Increasing the spatial coverage of soundings used in the generation of Level3 products will further decrease an already small relatively clear sky bias in the Level-3 products found in the Version-5 Level-3 products. The accuracy of AIRS Only Level-3 products would be slightly poorer than those using the AIRS/AMSU system, as expected, but would still be of very quality in the event that the AMSU-A instrument should eventually cease to produce useful observations.

Figure 2 shows that Version-6 retrievals using DA Quality Control thresholds are of comparable accuracy to Version-5 retrievals using Tight Quality Control thresholds but with a substantially greater yield, and Version-6 retrievals using Climate Quality Control thresholds have somewhat poorer RMS errors than Version-5 retrievals using Standard Quality Control near the surface, but with a substantially increased yield. Figures 3a and 3b compare the accuracy of Version-5 and Version-6 retrievals when evaluated on two common ensembles, an easy ensemble of cases and a difficult ensemble of cases. The solid lines in Figure 3 show yield and RMS errors using the easy ensemble of cases, which are the ensemble of Version-5 cases accepted using Version-5 Tight Quality Control thresholds. The dashed difficult ensemble of cases is the ensemble of Version-6 cases accepted using Version-6 Climate Quality Control thresholds. As before, red 


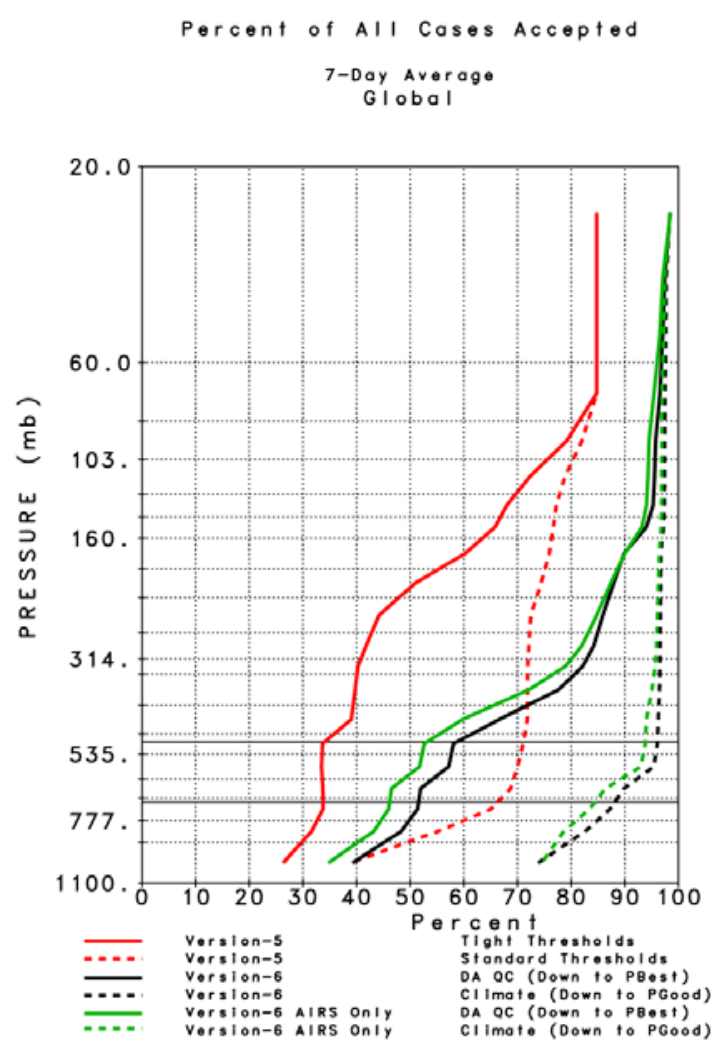

Fig. 2a

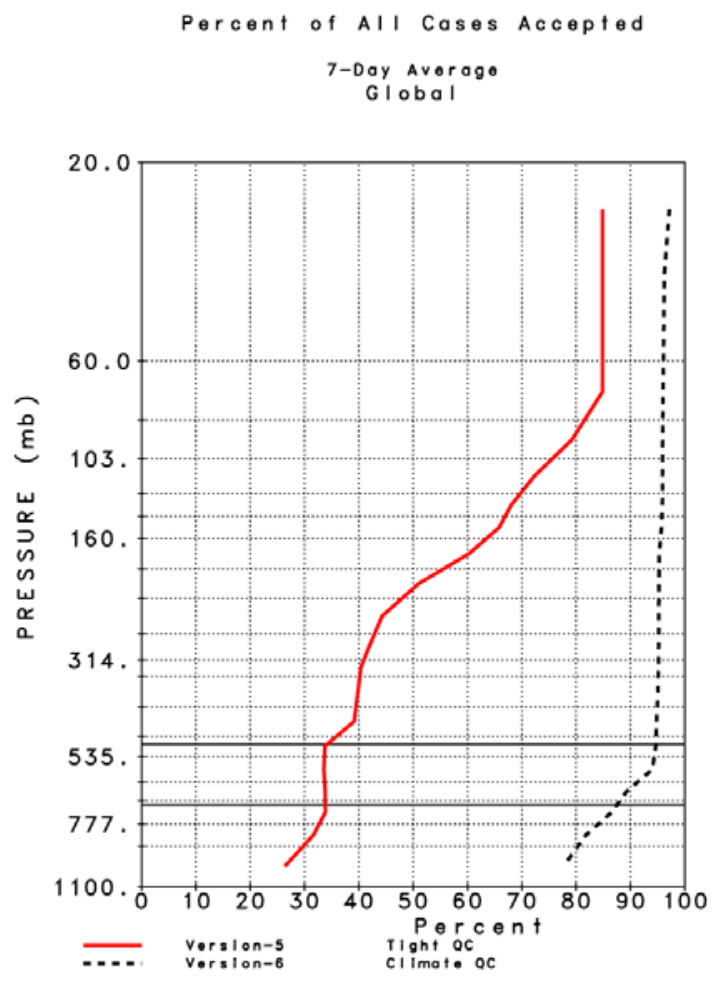

Fig. 3a

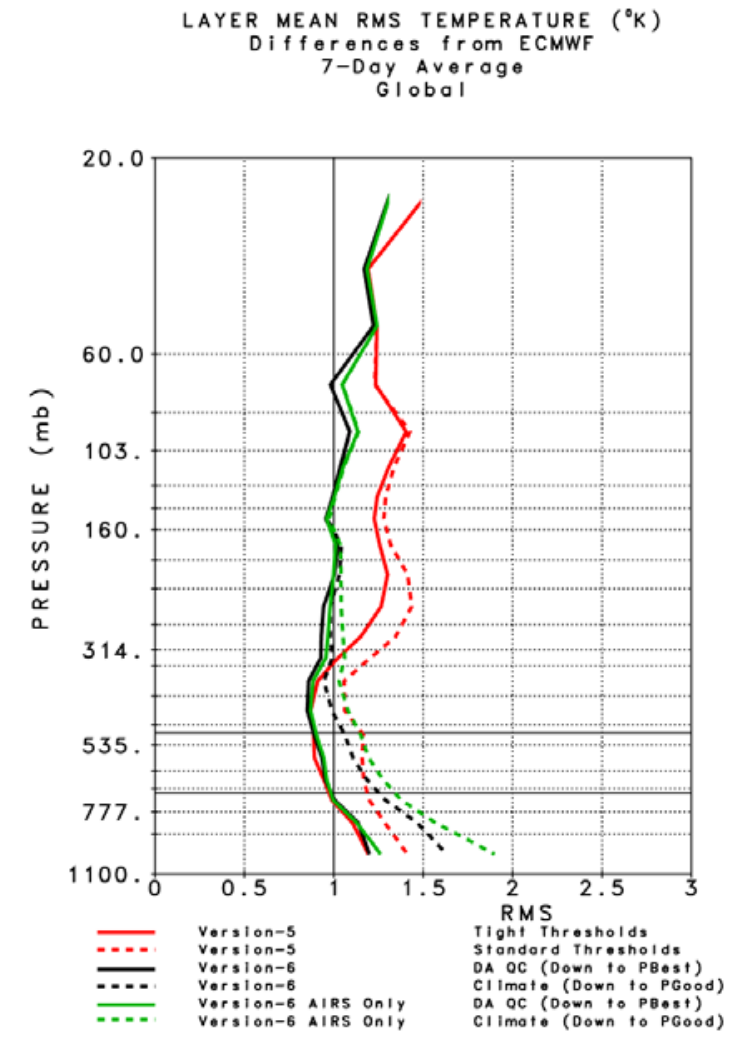

Fig. 2b

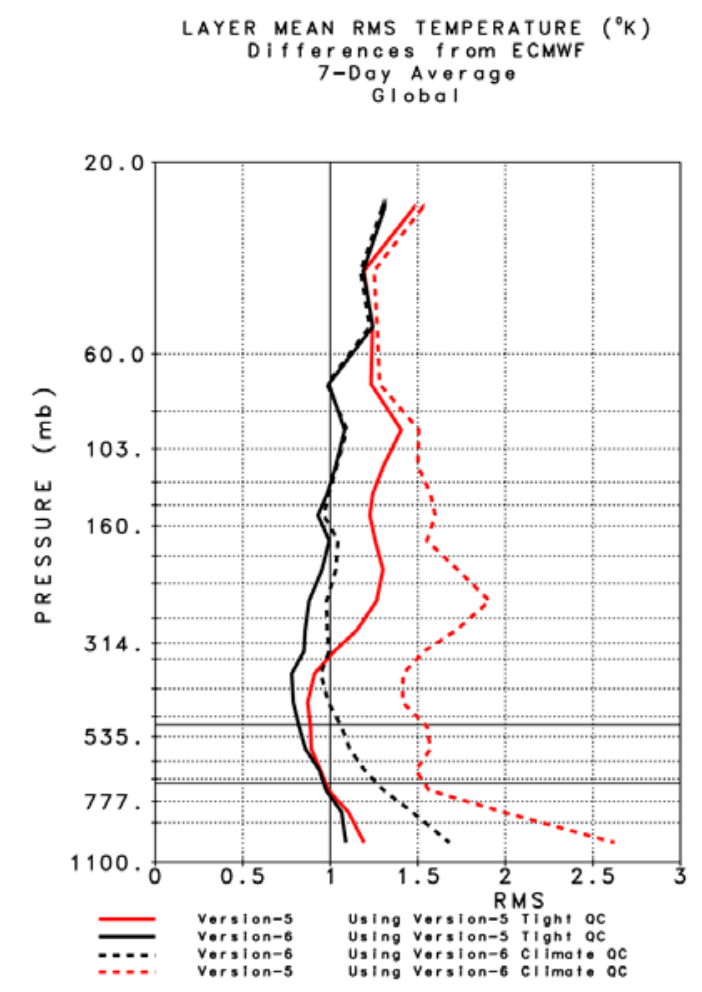

Fig. 3b 
shows results of Version-5 on these ensembles of cases and black shows results of Version-6 retrievals on these ensembles of cases. The two curves shown in Figure 3a match the same color curves shown in Figure 2a, and the solid red and dashed black curves shown in Figure 3b match those shown in Figure 2b. Figure 3b shows that when compared on common ensembles, Version-5 retrievals are poorer at all levels than Version-6 retrievals, and the degree of degradation increases significantly when compared using more difficult cases. Loosening the Version-5 thresholds to provide yields similar to that obtained using Version- 6 climate thresholds would have produced RMS errors that are too poor for use in climate studies. A significant contributor to this improvement is the new use of the neural network startup state in Version-6, which degrades much more slowly with increasing cloud cover than does the regression based start-up state used in Version-5. Another reason for the improved performance of Version-6 Quality Controlled temperature profiles compared to Version- 5 is the substantial improvement made in the Version- 6 determination of surface skin parameters, especially over land.

\subsection{Quality Controlled ocean surface skin temperature}

Version-6 and Version-5 both use the same Quality Control procedure with regard to ocean surface skin temperature (SST). In each version, values of ocean $T_{s}$ were considered acceptable if the error estimate $\Delta T_{s}$ was less than a threshold value $\delta T_{s}$. Figure 4a shows histograms of total seven day counts of Quality Controlled ocean SST retrieval as a function of SST differences from ECMWF "truth" obtained using Version-5, and Version-6 retrievals. The percent of all cases accepted, the standard deviation of the differences from ECMWF truth, and the percent outliers (errors more than 3K from the mean) are indicated in Figure 4a. Results for day and night are shown separately. Version-6 Quality Controlled SST retrievals have considerably higher yields with smaller standard deviations and similar or smaller percent outliers than the comparable Quality Controlled Version-5 SST's. The improvement is actually greater during the day than at night, even though values of Version-6 $T_{s}$ are determined using only shortwave window channels, which are highly sensitive to effects of solar radiation reflected by clouds and the surface, while in Version-5, $T_{s}$ was determined using both longwave and shortwave window channels.

Figure 4b shows the percent of all seven day SST cases $50^{\circ} \mathrm{N}-50^{\circ} \mathrm{S}$ accepted as a function of retrieved fractional cloud cover for both Version-5 and Version-6. Results are again shown separately for day and night cases. Version-5 Quality Control methodology automatically rejected all retrievals with fractional cloud cover greater than $90 \%$, both with regard to temperature profile and surface skin temperature. Version- 6 does not have this restriction. All cases shown in this figure were included in the statistics shown in Figure 4a. As shown in Figure 4a, Version-6 has a considerably higher percent yield than Version-5, with better overall accuracy, both under daytime and nighttime conditions. Version-6 Quality Controlled SST retrievals have higher yields than Version-5 at all cloud fractions both day and night. The relative improvement in the ability to determine accurate Quality Controlled SST's between Version-6 and Version-5 increases dramatically with increasing cloud cover. For example, at 50\% fractional cloud cover, Version-6 accepts roughly $30 \%$ of the cases during the day and $20 \%$ at night, while Version- 5 accepts roughly $8 \%$ of the cases under both day and night conditions. This increased yield under more difficult cloud conditions is significant for climate applications.

Figure 4c shows the RMS errors of Version- 5 and Version-6 accepted sea surface temperatures as a function of fractional cloud cover. RMS errors of accepted cases with fractional cloud cover greater than $80 \%$ for Version-5, and greater than $90 \%$ for Version-6, are not shown because in each case, the ensembles of accepted cases were very small. RMS errors of Version-6 SST retrievals grow slowly with increasing fractional cloud cover from about $0.65 \mathrm{~K}$ under clear conditions to about $1.4 \mathrm{~K}$ at $90 \%$ fractional cloud cover, with daytime and nighttime errors being very close to one another. Version- 6 SST errors at a given cloud fraction are better than or comparable to those of Version-5, with significantly higher yields, especially at night.

\subsection{Quality Controlled surface spectral emissivities}

In Version-5, both longwave and shortwave surface spectral emissivities are solved for simultaneously with each other, as well as with $T_{s}$, using observations in both longwave and shortwave window channels. In Version-6, the shortwave spectral emissivity is solved for, together with $T_{s}$, using only shortwave window channel observations, and the longwave spectral emissivity is solved for in a subsequent step using only longwave window observations. This procedure 


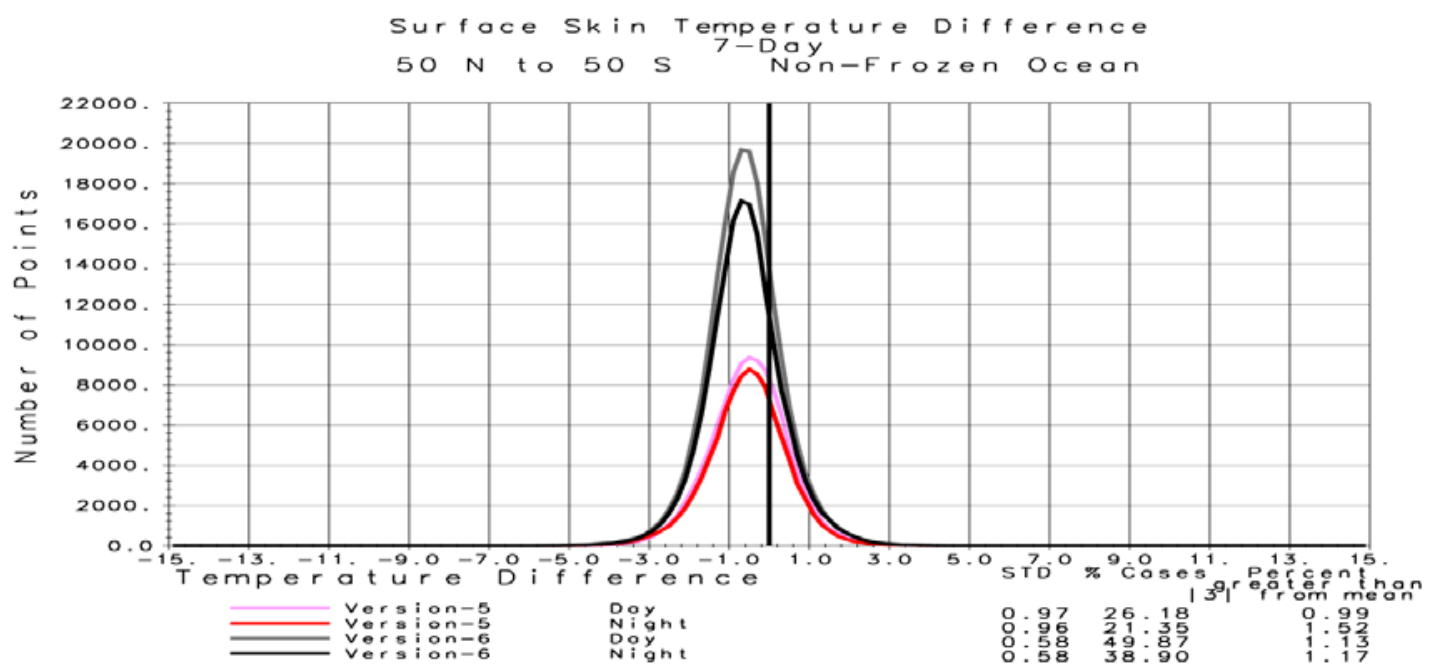

Fig. 4a

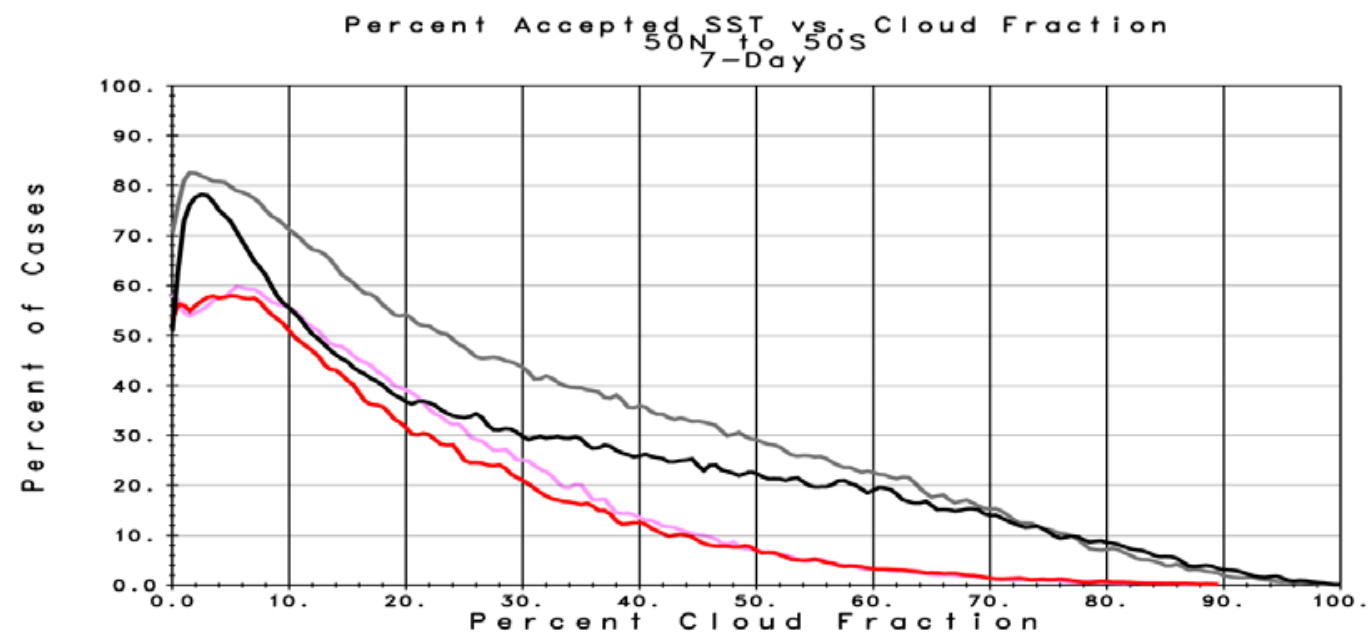

Fig. 4b

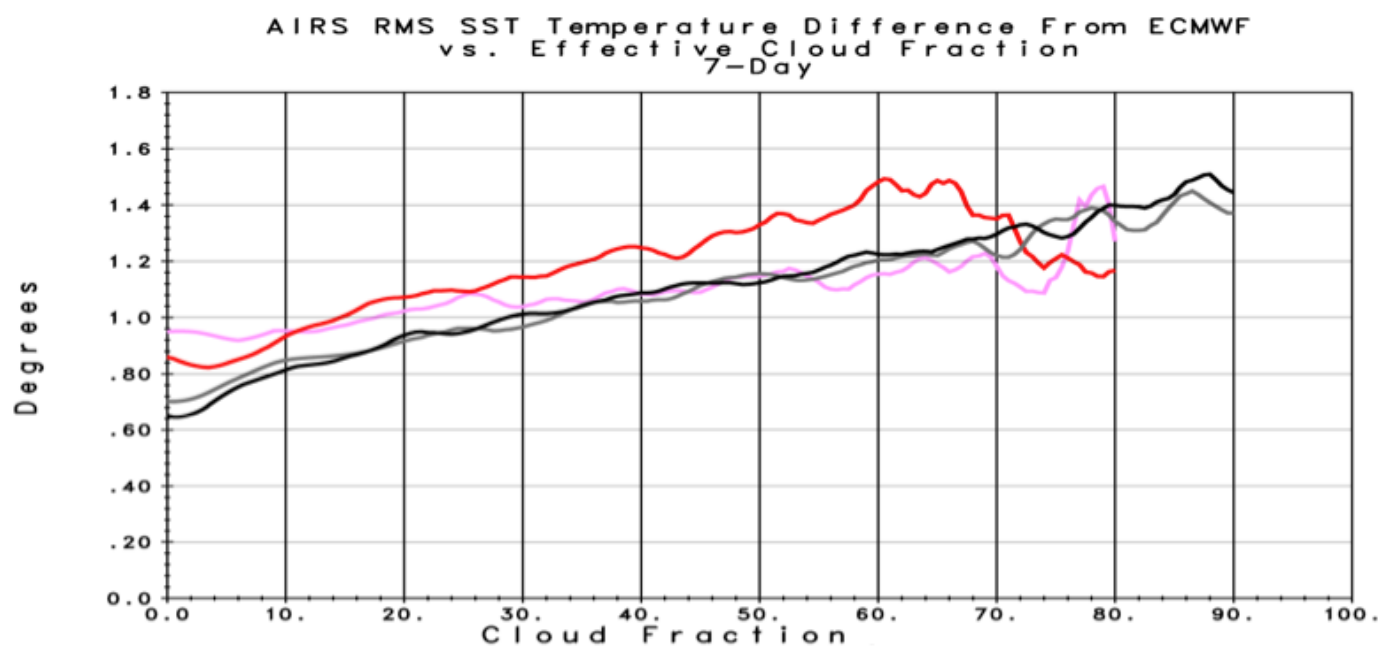

Fig. 4c 

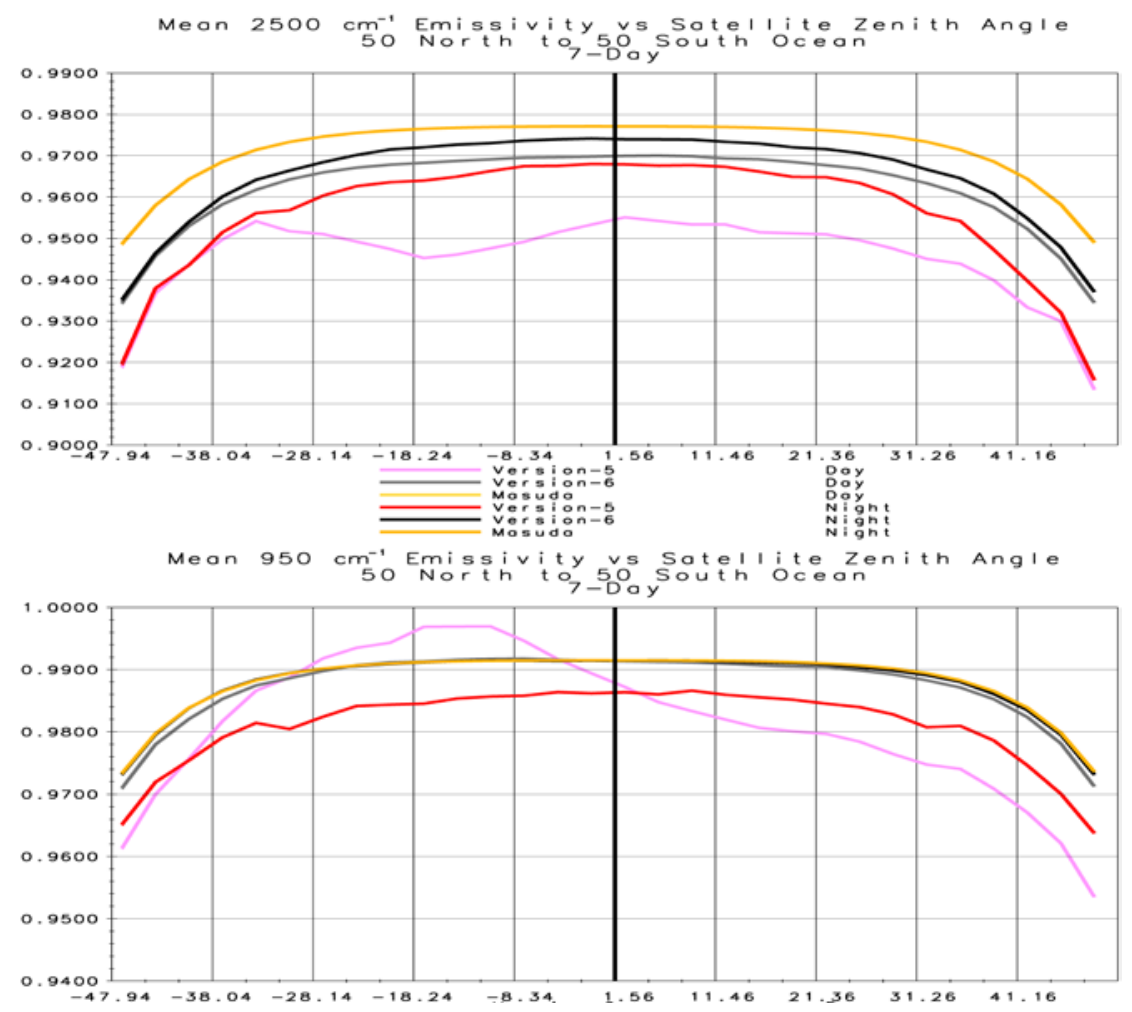

Fig. 5

improves not only retrieved values of $T_{s}$, but of the surface spectral emissivities as well. Over ocean, surface spectral emissivity is well described by the Masuda model which parameterizes ocean surface spectral emissivity as a function of frequency, viewing angle, and windspeed. Figure 5 shows seven day mean retrieved Quality Controlled values of Version-5 and Version-6 ocean surface spectral emissivity as a function of satellite zenith angle at two frequencies, 950 $\mathrm{cm}^{-1}$ (longwave) and $2500 \mathrm{~cm}^{-1}$ (shortwave). Also shown are values of the Masuda model, evaluated at a windspeed of five $\mathrm{m} / \mathrm{sec}$. The Masuda model is not perfect even at the correct wind speed, and the assumption of a wind speed of five $\mathrm{m} / \mathrm{sec}$ is not representative of all cases. Nevertheless, the extent of agreement of retrieved ocean emissivities with the Masuda model gives a reasonable assessment of the accuracies of the retrievals. Version-6 ocean surface emissivities are much closer in the mean to Masuda than those using Version-5, especially at $950 \mathrm{~cm}^{-1}$.

Daytime and nighttime ocean surface spectral emissivities are expected to be very close to each other, and both sets of emissivities are expected to be symmetric with respect to satellite zenith angle. Daytime Version-5 surface emissivity shows a strong spurious feature centered near $-18^{\circ}$ satellite zenith angle at both frequencies. This is the satellite zenith angle that is most sensitive to the effect of sunglint reflected by the ocean surface. The fact that this artifact occurs at $2500 \mathrm{~cm}^{-1}$, which is sensitive to reflected solar radiation, is understandable though undesirable. This spurious feature in daytime Version-5 spectral emissivity shows that the Version-5 retrieval algorithm doesn't handle effects of solar radiation reflected by the earth's surface adequately. The same spurious feature also shows up in retrieved Version-5 surface emissivity at $950 \mathrm{~cm}^{-1}$, which is a frequency at which satellite observations are not affected directly by reflected solar radiation. This spurious feature at $950 \mathrm{~cm}^{-1}$ is a result of the simultaneous solution of both longwave and shortwave ocean surface emissivity in Version-5, using both longwave and shortwave ocean window observations. This spurious feature does not occur in retrieved Version-6 ocean surface emissivity at either frequency.

Figure 5 shows mean values of retrieved ocean surface emissivity. Figure 6 shows the standard deviation of the retrieved emissivities as a function of satellite zenith angle. Standard deviations of retrieved Version-6 surface emissivities are considerably smaller than those of Version-5 surface emissivities at both frequencies, especially at zenith angles sensitive to sunglint. This is indicative that the Version-6 retrieval algorithm is not only more accurate than Version-5, but it is much more stable as well. 


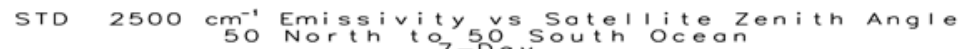
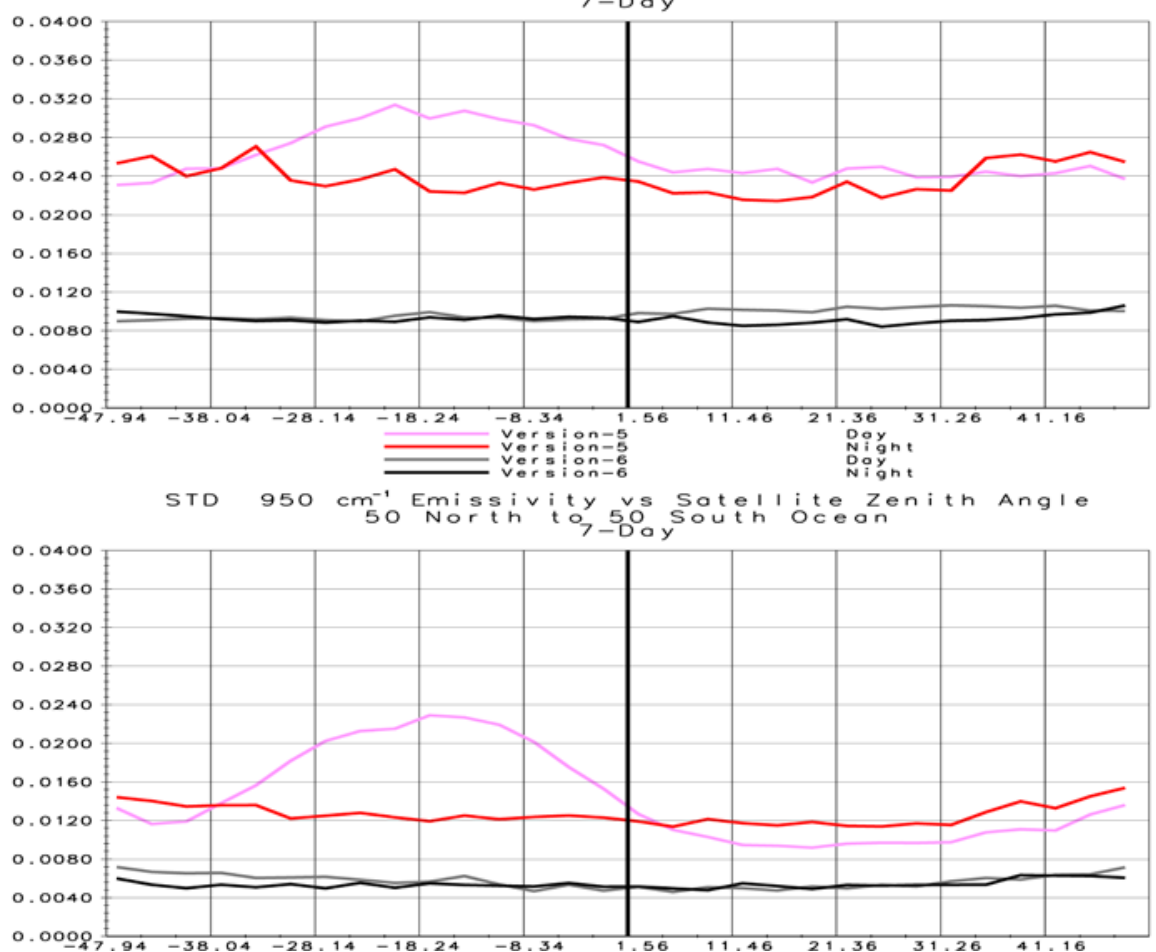

Fig. 6

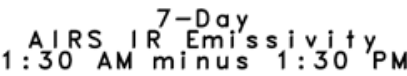

version-6
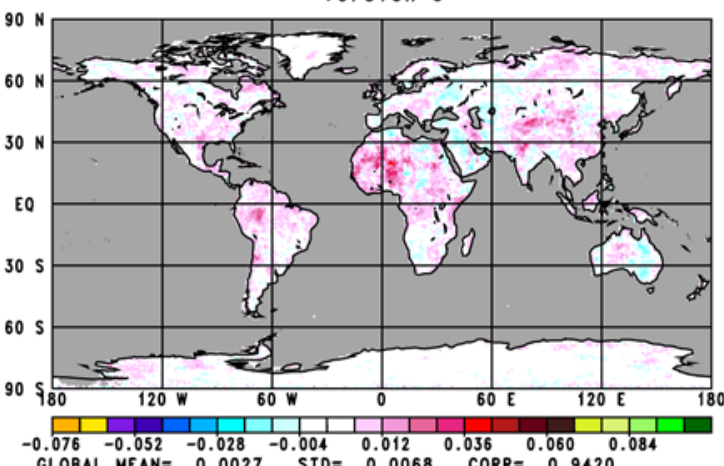

GLOBAL MEAN $=0.0027$ STD $=0.0068$ CORR $=0.9420$

vers $12 \operatorname{cm}^{-1}-5$

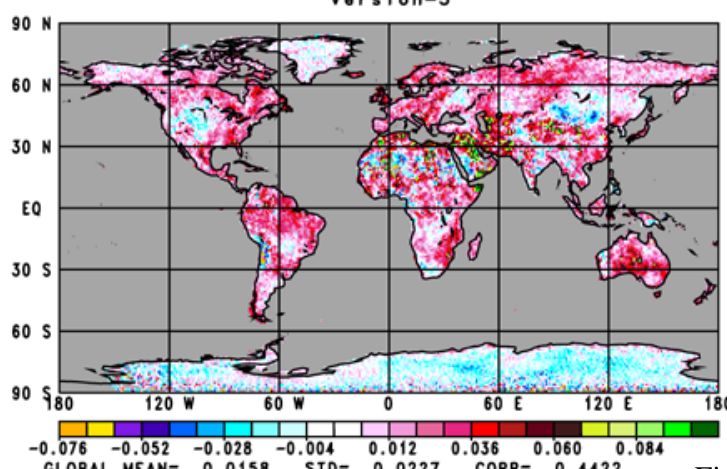

$2500 \mathrm{~cm}^{-1}$
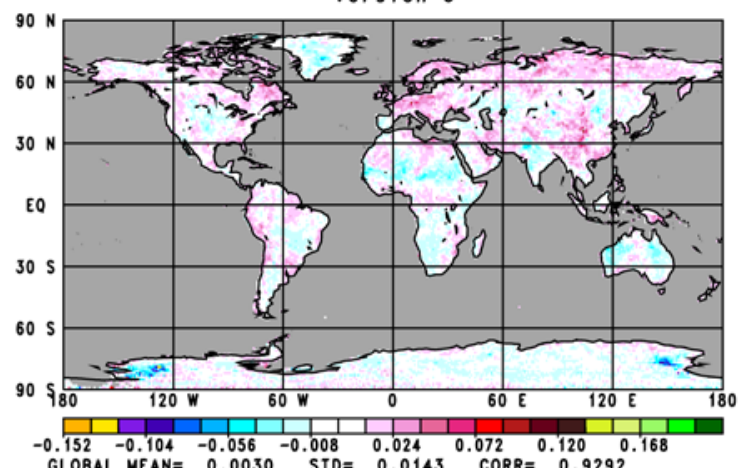

GLOBAL MEAN $=0.0030$ STD $=0.0143$ CORR $=0.9292$

Vors $2500 \mathrm{~cm}^{-1}$

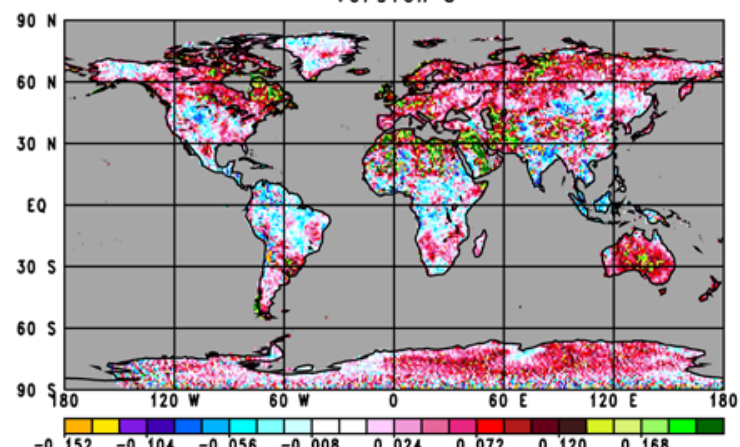

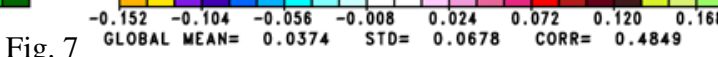


Surface spectral emissivity is much more variable over land than it is over ocean, both in space and in time of year as it depends on surface characteristics such as vegetation cover and snow cover. Nevertheless, at a given location and time of year, the land surface emissivity is expected to have very small day/night differences. Figure 7 shows seven day mean values of 1:30 AM minus 1:30 PM land emissivities at both $950 \mathrm{~cm}^{-1}$ and $2500 \mathrm{~cm}^{-1}$ obtained using the Version-6 retrieval algorithm and the Version-5 retrieval algorithm. As found with regard to ocean surface spectral emissivity, the day/night differences of Version-6 land surface emissivities are considerably smaller than those obtained using Version5. This indicates that the Version-6 retrieval methodology is performing much better than Version-5 over land as well. This figure also provides indirect evidence that day/night land surface skin temperature differences obtained using Version-6are superior to those obtained using Version-5.

\section{SUMMARY}

This paper shows that improved AIRS Quality Controlled soundings of atmospheric temperature profile $T(p)$, ocean surface skin temperature $T_{s}$ and ocean and land surface spectral reflectivities are obtained by use of cloud cleared radiances $\hat{R}_{i}$ of only shortwave window channels in the determination of $T_{s}$, and primarily of $4.2 \mu \mathrm{m} \mathrm{CO}_{2}$ channels in the determination of $T(p)$. Observed radiances $R_{i}$ are used in the longwave $15 \mu \mathrm{m} \mathrm{CO}_{2}$ band and $11 \mu \mathrm{m}$ window in the

determination of $\hat{R}_{i}$ for all channels. This improvement in sounding capability exists for relatively clear situations, and is magnified with increasing cloud cover. Results obtained during the day are at least as good, if not better, than those obtained at night. Similar results are obtained in the Version-6 AIRS Only system.

This improved sounding methodology is made possible as a result of a few factors, both theoretical and instrumental. First of all, use of improved radiative transfer physics allows for the accurate computation of the effects of non-LTE on the observed radiances in the $4.2 \mu \mathrm{m} \mathrm{CO}$ band during the day. Secondly, the cloud clearing methodology used to analyze AIRS data removes the effects of solar radiation reflected by clouds from the clear column radiances $\hat{R}_{i}$ used to generate the solution. Effects of solar radiation reflected by the surface on the shortwave radiances are accounted for directly as part of the surface parameter retrieval process. Also, improved error estimates and Quality Control methodology allows for the screening of cases in which aspects of the retrieval methodology may have performed poorly. Version-6 temperature profile retrievals also benefit from the use of a start-up state generated using neuralnetwork methodology. ${ }^{7,8}$ This approach allows for accurate retrievals under more stressing cloud conditions.

\section{REFERENCES}

[1] Pagano, T. S., Aumann, H. H., Hagan, D. E. and Overoye, K., "Prelaunch and in-flight radiometric calibration of the Atmospheric Infrared Sounder (AIRS),” IEEE Trans. Geoscience and Remote Sensing, 41, 265-273, DOI: 10.1109/TGRS.2002.808324 (2003).

[2] Susskind, J, Blaisdell, J.M., Iredell, L. and Keita, F., "Improved temperature sounding and quality control methodology using AIRS/AMSU data: The AIRS Science Team Version-5 Retrieval Algorithm,” IEEE Trans. on Geoscience and Remote Sensing, Issue: 99, DOI: 10.1109/TGRS.2010.2070508, 1-15 (2011).

[3] Susskind, J., Barnet, C.D., and Blaisdell, J.M., "Retrieval of atmospheric and surface parameters from AIRS/AMSU/HSB data in the presence of clouds,” IEEE Trans. Geoscience and Remote Sensing, 41, 390-409, DOI: 10.1109/TGRS.2002.808236 (2003).

[4] Susskind, J., Barnet, C., Blaisdell, J., Iredell, L., Keita, F., Kouvaris, L., Molnar, G. and Chahine, M., “Accuracy of geophysical parameters derived from Atmospheric Infrared Sounder/Advanced Microwave Sounding Unit as a function of fractional cloud cover,” J. Geophys. Res., 111, D09S17, DOI:10.1029/2005JD006272 (2006).

[5] Chahine, M. T., "Remote sensing of cloudy atmospheres. II. Multiple cloud formations,” J. Atmos. Sci., 34, 744-757 (1977).

[6] Chahine, M. T., "Remote sensing of cloudy atmospheres. I. The single cloud layer,” J. Atmos. Sci., 31, 233-243 (1974).

[7] Tao, Z., Blackwell, W.J., Staelin, D.H., "Variance estimation of individual geo-physical parameter retrievals," IEEE Trans. Geoscience and Remote Sensing, submitted for publication (2011).

[8] Blackwell, W.J., "Neural network Jacobian analysis for high-resolution profiling of the atmosphere," EURASIP Journal on Advances in Signal, submitted for publication (2011). 